\title{
Scaling of Chiral Order Parameter in Two-Flavor QCD
}

\author{
Y. Iwasaki, ${ }^{1,2}$ K. Kanaya, ${ }^{1,2}$ S. Kaya, ${ }^{1}$ and T. Yoshié ${ }^{1,2}$ \\ ${ }^{1}$ Institute of Physics, University of Tsukuba, Ibaraki 305, Japan \\ ${ }^{2}$ Center for Computational Physics, University of Tsukuba, Ibaraki 305, Japan
}

(Received 9 September 1996)

\begin{abstract}
The finite temperature transition of QCD with two degenerate light quarks is studied on the lattice with a renormalization group improved gauge action and the Wilson quark action. We have made simulations on an $8^{3} \times 4$ lattice near the chiral transition point. It is shown that the chiral condensate, which is the order parameter of chiral symmetry, satisfies remarkably a scaling relation with the exponents of the three-dimensional $O(4)$ Heisenberg model. This indicates that the chiral transition in two-flavor QCD is of second order in the continuum limit. [S0031-9007(96)02164-3]

PACS numbers: 12.38.Gc, 11.30.Rd
\end{abstract}

Understanding the nature of the finite temperature transition in QCD with two degenerate light quarks $\left(N_{F}=2\right)$ is an important step toward the clarification of the transition in the real world. It is plausible from an argument based on an effective $\sigma$ model [1] that, if the chiral transition (transition in the chiral limit) is of second order, QCD with two flavors belongs to the same universality class as the three-dimensional $O(4)$ Heisenberg model. This universality provides us with several useful scaling relations that can be confronted with numerical results. These scaling relations were first tested on the lattice for staggered quarks [2], where some evidence consistent with the $O(4)$ scaling was reported.

In this paper, we study scaling behavior of the QCD transition with two degenerate light quarks on the lattice using the Wilson formalism of fermions, and compare the result with a conjectured scaling relation. The Wilson formalism of fermions is the only known lattice formalism which possesses a local action for any number of flavors. Therefore, it is important to investigate the scaling behavior with Wilson quarks and compare the results with those for staggered quarks for which the action is nonlocal for two flavors.

We use a renormalization group improved gauge action [3] which is expected to be closer to the renormalized trajectory compared with the standard one-plaquette gauge action, and therefore is expected to show scaling behavior on a coarse lattice.

The continuum limit does not depend on the choice of the action if the action belongs to the same universality class. However, the way of the approach to the continuum limit does depend on the choice of the action. When one uses the standard one-plaquette gauge action and the Wilson quark action for a study of QCD with two flavors at finite temperatures, one encounters the existence of severe lattice artifacts on lattices with the lattice size in the euclidean time direction $N_{t}=4$ and 6 [4-6]. Note the temperature on the lattice is given by $T=1 / a N_{t}$, with $a$ being the lattice spacing. A way out of these lattice artifacts with the standard action is to increase $N_{t}$ in order to further approach toward the continuum limit. However, our previous study on an $18^{3} \times 24$ lattice suggests that $N_{t}>18$ is required to have the chiral transition in the scaling region [7]. It is difficult to perform simulations on such a large lattice even with a powerful computer of today. Therefore, we instead adopt a renormalization group improved gauge action and the Wilson quark action.

Before going into the discussion of the present work, let us first describe the unexpected phenomena observed by the MILC collaboration with the standard gauge action. The transition is smooth for both heavy and light quarks, while the transition is very sharp at $N_{t}=4$ (even a first order transition at $N_{t}=6$ ) in the range of intermediate quark masses $(\beta \simeq 5.0$ ) [4]. This is completely different from what is supposed to be realized in the continuum limit: As the quark mass increases from the chiral limit, the transition becomes weaker, and it becomes strong again only when the quark mass is heavy enough to recover the first order transition of the SU(3) gauge theory. They also observed a cusp in the behavior of $m_{\pi}^{2}$ in terms of $1 / K$ with $\beta$ fixed ( $m_{\pi}$ is the screening pion mass and $K$ is the hopping parameter). Furthermore, at $\beta \lesssim 5.3$, the value of the quark mass $m_{q}$, defined through an axial-vector Ward identity [8,9], depends on the phase of the system and shows unexpected $1 / K$ dependence with $\beta$ fixed in the high temperature phase $[4,10]$, which is in sharp contrast with the case of $\beta \gtrsim 5.5$, where it does not depend on the phase [11]. In the following, we show that these unexpected phenomena are removed by improving the lattice action.

Now let us discuss the present work. The renormalization group improved gauge action $S_{q}^{\mathrm{IM}}$ we use is given by

$$
S_{g}^{\mathrm{IM}}=\frac{1}{g^{2}}\left\{c_{0} \sum(1 \times 1 \text { loop })+c_{1} \sum(1 \times 2 \text { loop })\right\},
$$

with $c_{1}=-0.331$ and $c_{0}=1-8 c_{1}$ [3]. Here the loops in the sums are defined by the trace of the ordered product of link variables, and each oriented loop appears once in the sum. 
We mainly perform simulations for $N_{t}=4$ with spatial lattice size $8^{3}$ at $\beta \equiv 6 / g^{2}=1.0-4.0$ near the finite temperature transition point. We use an antiperiodic boundary condition in the $t$ direction and periodic boundary conditions otherwise. The configurations are updated using the hybrid Monte Carlo method with the molecular dynamics time step size $\delta \tau=0.01$ (except for a simulation point $\beta=1.5, K=0.206$ on the $N_{t}=4$ lattice where $\delta \tau=0.002$ ). We perform simulations of about $100-1300$ trajectories after thermalization for each set of parameters, $\beta$ and $K$. Errors are estimated by the jackknife method. A preliminary report is given in Ref. [10].

Our results for the phase diagram are shown in Fig. 1. The chiral line $K_{c}$ [6] is defined by the vanishing point of the screening pion mass, $m_{\pi}=0$, on an $8^{4}$ lattice (doubled in the $z$ direction for the spectrum calculation). From the values of $m_{\rho}$ on the $K_{c}$ line, we estimate $a^{-1}=838(20), 889(31), 979(60)$, and 1108(33) MeV at $\beta=1.5,1.7,1.9$, and 2.1 , respectively. Although the lattices are coarse, we expect scaling behavior of physical quantities because of the improvement of the action, as mentioned earlier. The finite temperature transition/ crossover line $K_{t}$ for $N_{t}=4$ is determined on the $8^{3} \times 4$ lattice from the condition that $P=0.10(2)$, where $P$ is the Polyakov line expectation value. This condition corresponds to the criterion that the crossover point is identified as the peak position of the susceptibility of the Polyakov loop, although our limited statistics sometimes make the peak of the susceptibility not so clear. We also note that this is consistent with the criterion that the crossover points are identified from rapid changes of physical quantities (see Fig. 2). We identify the crossing point of the $K_{c}$ and $K_{t}$ lines as the point of the finite temperature chiral transition point, $\beta_{\mathrm{ct}}$. We refer to Secs. 3 and 7 of Ref. [6] for discussion concerning the

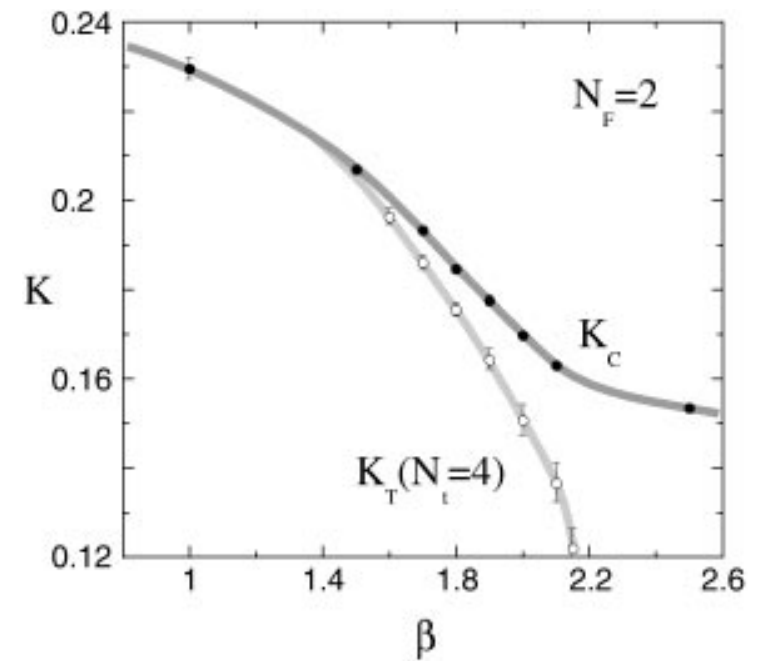

FIG. 1. Phase diagram for $N_{F}=2$ QCD with the renormalization group improved gauge action, Eq. (1), and the Wilson quark action. The lines are to guide the eyes. existence of the crossing point. Note that the $K_{c}$ line is defined at zero temperature. Applying the "on- $K_{c}$ method" [6] (monitoring the number of iterations for the quark matrix inversion along the $K_{c}$ line), we conclude that the point $\beta=1.3$ belongs to the confining phase in the chiral limit.

For the case of the standard gauge action, we pointed out that the simulation points where the strong transitions mentioned earlier are observed on the $K_{t}$ line [4] are just those where the $K_{t}$ line approaches toward the $K_{c}$ line after initially deviating from it due to the crossover phenomenon between weak and strong coupling regions of QCD, and that therefore it seems plausible that the strong transition is a result of lattice artifacts caused by this unusual relation of the $K_{t}$ and $K_{c}$ lines [5,6,10].

Unlike for the case of the standard gauge action, the distance between the $K_{t}$ and $K_{c}$ lines shown in Fig. 1 grows monotonically when we increase $\beta$ from the chiral transition point $\beta_{\mathrm{ct}} \sim 1$.4. In accordance with this, the transition becomes monotonically weaker with $\beta$ as shown in Fig. 2(a) for the Polyakov loop. This is in sharp contrast with the case of the standard gauge action. Note that the change of the Polyakov loop at the finite temperature transition/crossover is very smooth for a wide range of $\beta$. The change of $m_{\pi}^{2}$ is also smooth for larger values of $\beta$, and it becomes sharper as $\beta$ decreases [cf. Fig. 2(b)]. The straight line envelope of $m_{\pi}^{2}$ in the high temperature phase $\left(N_{t}=4\right)$ agrees with $m_{\pi}^{2}$ in the low temperature

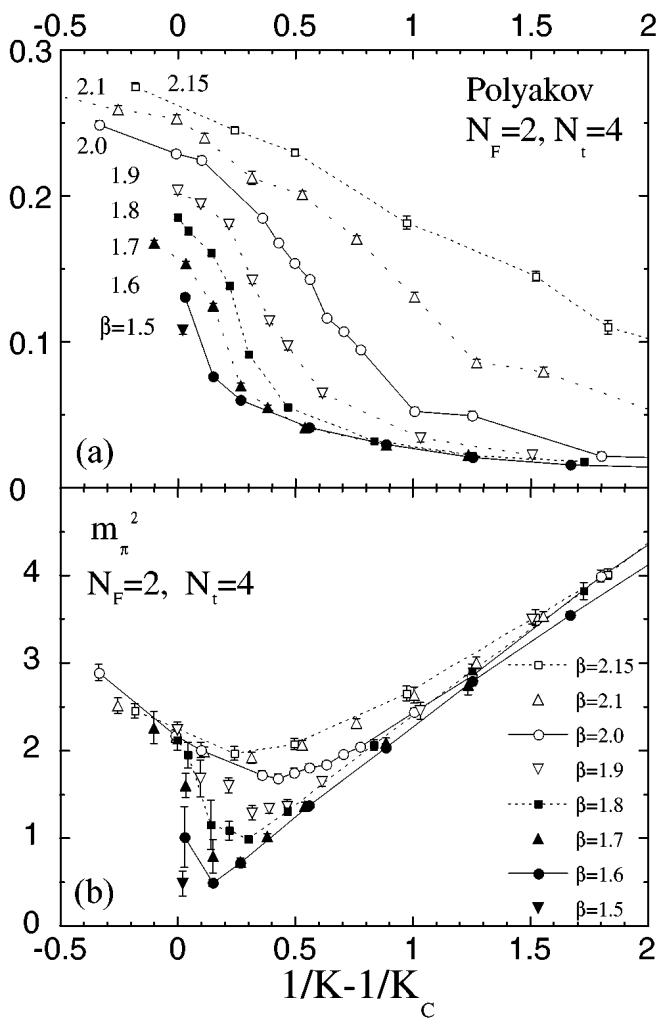

FIG. 2. (a) The Polyakov loop and (b) the pion screening mass. 
phase $\left(N_{t}=8\right)$, and corresponds to the partially conserved axial-vector current relation $m_{\pi}^{2} \propto m_{q}$. The smoothness of physical observables strongly suggests that the transition is a crossover at $\beta>\beta_{\mathrm{ct}}$.

It should be noted that the value of $m_{\pi}^{2}$ on the $K_{c}$ line monotonically decreases to zero as $\beta \rightarrow \beta_{\mathrm{ct}}+0$ (cf. Fig. 3), suggesting that the chiral transition is continuous. We also find that $m_{\pi}^{2}$ on the $K_{t}$ line shows a similar monotonic decrease [10]. It might be emphasized that the value of $m_{q}$ in the high temperature phase agrees well with that in the low temperature phase and does not show the strange behavior mentioned before for the case of the standard action (see Fig. 4).

These nice properties, which are in accordance with naive expectations, encourage us to begin a scaling study with Wilson quarks.

From the universality argument, we expect that the magnetization $M$ near the second order phase transition point can be described by a single scaling function,

$$
M / h^{1 / \delta}=f\left(t / h^{1 / \beta \delta}\right),
$$

where $h$ is the external magnetic field and $t=[T-$ $\left.T_{c}(h=0)\right] / T_{c}(h=0)$ is the reduced temperature. For three-dimensional $O(4)$ models, the critical exponents in (2) are given by $1 /(\beta \delta)=0.537(7)$ and $1 / \delta=$ $0.2061(9)$ [12]. In QCD, $h$ corresponds to the quark mass and $M$ corresponds to the chiral condensate. DeTar tested this scaling for the case of two-flavor staggered quarks [13] and reported that the data are consistent with $O(4)$ and $O(2)$ scaling.

For Wilson quarks, the naive definition of $\langle\bar{\Psi} \Psi\rangle$ for the chiral condensate is not adequate because the chiral symmetry is explicitly broken due to the presence of the Wilson term. A proper subtraction and a renormalization are required to obtain the correct continuum limit. A properly subtracted $\langle\bar{\Psi} \Psi\rangle$ can be defined via an axialvector Ward identity [8],

$$
\langle\bar{\Psi} \Psi\rangle_{\text {sub }}=2 m_{q} a Z \sum_{x}\langle\pi(x) \pi(0)\rangle,
$$

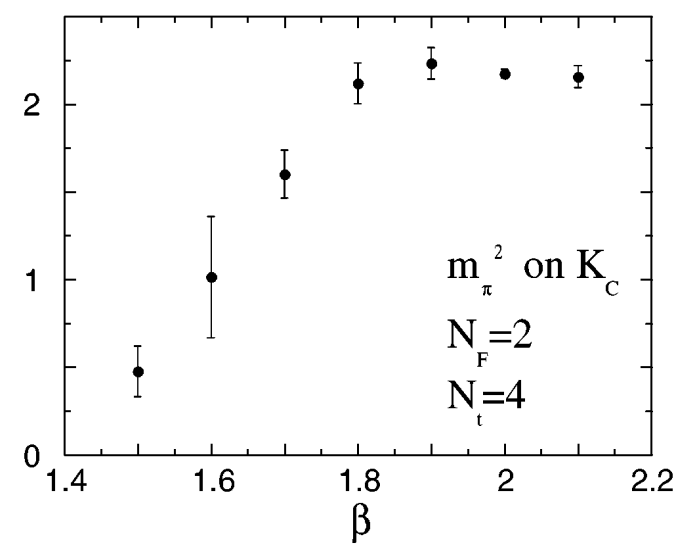

FIG. 3. $m_{\pi}^{2}$ on $K_{c}$. where $Z$ is the renormalization coefficient. This definition is consistent with the identification of the magnetization in Ref. [1]. $\langle\bar{\Psi} \Psi\rangle_{\text {sub }}$ was shown to have a nonvanishing value in the chiral limit in the confining phase of quenched QCD [14]. For our purpose, it is enough to use the tree value: $Z=(2 K)^{2}$. Our results of $\langle\bar{\Psi} \Psi\rangle_{\text {sub }}$ for $N_{t}=4$ are shown in Fig. 5 .

If the two-flavor QCD belongs to the same universality class as three-dimensional $O(4)$ spin models, the chiral condensate should satisfy the scaling relation (2) with the identification $M=\langle\bar{\Psi} \Psi\rangle_{\mathrm{sub}}, h=2 m_{q} a$, and $t=\beta-$ $\beta_{\mathrm{ct}}$. We further expect that the scaling function $f(x)$ itself is a universal function [15] because $f(x)$ is determined by the universal singular structure of the free energy around the UV fixed point. We make a fit to the scaling function recently obtained for an $O(4)$ model [15], by adjusting $\beta_{\text {ct }}$ and the scales for $t$ and $h$, with the exponents fixed to the $O(4)$ values. Figure 6(a) shows our result with $\chi^{2} / d f=0.61$. The scaling ansatz works remarkably well with the $O(4)$ exponents. The resulting $\beta_{\mathrm{ct}}=$ $1.35(1)$ is slightly smaller than the value $\simeq 1.4$ obtained by linear extrapolations of the $K_{t}$ line in the $(\beta, K)$ space (cf. Fig. 1), the $m_{\pi}^{2}$ on $K_{c}$ (cf. Fig. 3), and also the $m_{\pi}^{2}$ on $K_{t}$ [10]. However, the $O(4)$ universality predicts [1] that $\beta_{c}\left(m_{q}\right)-\beta_{\mathrm{ct}} \propto m_{q}^{1 / \beta \delta}$, and $m_{\pi}^{2} \sim\left(\beta_{c}-\beta_{\mathrm{ct}}\right)^{\gamma}$ on the $K_{c}$ and $K_{t}$ lines with $\gamma \simeq 1.4$, which implies that these lines should bend slightly near the chiral transition point to give a smaller $\beta_{\mathrm{ct}}$. Therefore, we conclude that $\beta_{\mathrm{ct}}=1.35(1)$ is consistent with the data.

Our data are limited to the region $t>0$ and on the lattice of $8^{3} \times 4$. We reserve the study in the case of small quark masses with $\beta$ closer to $\beta_{\mathrm{ct}}$ which requires a larger lattice, as well as that in the region $t<0$, for future investigations.

Finally, we test the mean-field (MF) exponents $[1 / \beta \delta=2 / 3,1 / \delta=1 / 3]$ suggested in Ref. [16]. We find that it is more difficult to arrange a wide range of data on a universal curve by adjusting $\beta_{\mathrm{ct}}$. A fit with the minimum $\chi^{2} / d f=2.5$ with the MF scaling function is obtained with $\beta_{\mathrm{ct}}=1.54(1)$. However, the data at

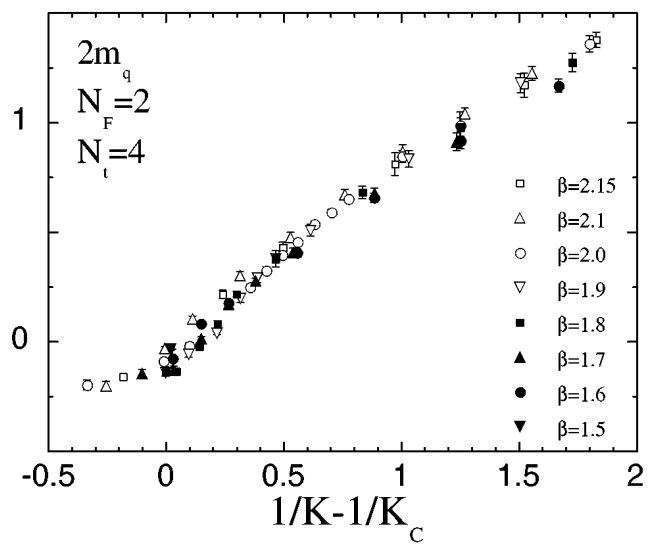

FIG. 4. The same as Fig. 2 for twice the quark mass. 


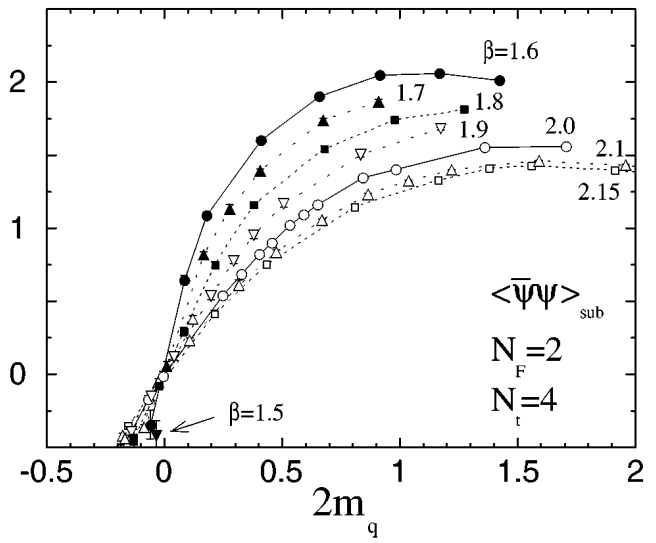

FIG. 5. Subtracted chiral condensate $\langle\bar{\Psi} \Psi\rangle_{\text {sub }}$ as a function of $2 m_{q} a$.

$\beta=1.6$ are completely off the fit. Furthermore, this value of $\beta_{\mathrm{ct}}$ is in conflict with the fact that the data of $m_{\pi}^{2}$ on the $K_{c}$ line indicates $\beta_{\mathrm{ct}} \leq 1.5$ (cf. Fig. 3). Restricting $\beta_{\mathrm{ct}} \leq 1.5$, we obtain the fit shown in Fig. 6(b) with $\chi^{2} / d f=3.3$. In contrast with the case of the $O(4)$ exponents, the MF scaling function cannot well reproduce the $t / h^{1 / \beta \delta}$ dependence of the data at fixed $\beta$, especially at small $\beta$ 's. As a result, all of the data

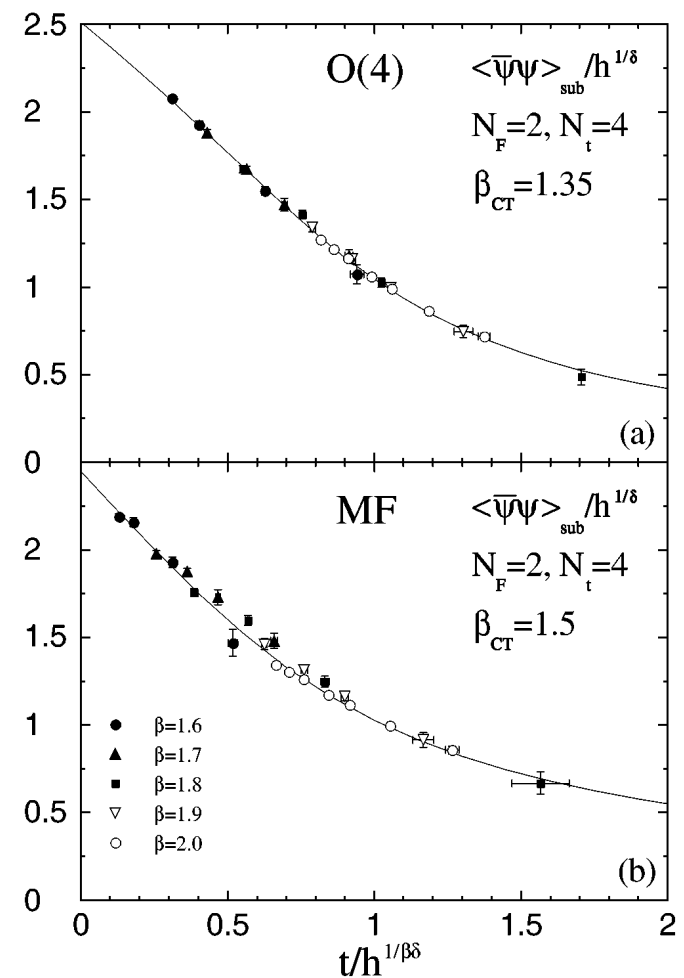

FIG. 6. Best fits to the scaling function with (a) $O(4)$ and (b) MF exponents (see text). The plot contains all the data shown in Fig. 5 within the range $0<2 m_{q} a<0.8$ and $\beta \leq$ 2.0. Solid curves are scaling functions obtained in an $O(4)$ spin model [15] and by a MF calculation, respectively. are more scattered than the case of the $O(4)$ exponents. When we decrease $\beta_{\mathrm{ct}}$ less than 1.4, which seems natural from other data (cf. Figs. 1 and 3), $\langle\bar{\Psi} \Psi\rangle_{\text {sub }} / h^{1 / \delta}$ does not fall on an approximate universal curve. Therefore, we conclude that the data do not favor the MF scaling.

The success of this scaling test with the $O(4)$ exponents strongly suggests that the chiral transition is of second order in the continuum limit. It also indicates that, with the improved gauge action, the chiral violation due to the Wilson term is sufficiently weaker than that introduced by the nonvanishing $m_{q}$, at least for $m_{q}$ 's at $\beta$ 's studied here. To strengthen the conclusion, a direct extraction of each critical exponent would be desirable.

We are grateful to Doug Toussaint for very useful discussions and sending us the data of the scaling function for an $O(4)$ spin model. The simulations are performed with Fujitsu VPP500/30 at the University of Tsukuba. This work is in part supported by the Grantin-Aid of Ministry of Education, Science and Culture (No. 07NP0401, No. 07640375, and No. 07640376).

[1] R. Pisarski and F. Wilczek, Phys. Rev. D 29, 338 (1984); F. Wilczek, Int. J. Mod. Phys. A 7, 3911 (1992); K. Rajagopal and F. Wilczek, Nucl. Phys. B399, 395 (1993).

[2] F. Karsch, Phys. Rev. D 49, 3791 (1994); F. Karsch and E. Laermann, ibid. 50, 6954 (1994).

[3] Y. Iwasaki, Nucl. Phys. B258, 141 (1985); Report No. UTHEP-118, 1983 (unpublished).

[4] C. Bernard et al., Phys. Rev. D 49, 3574 (1994); ibid. 46, 4741 (1992); T. Blum et al., ibid. 50, 3377 (1994).

[5] Y. Iwasaki, Nucl. Phys. (Proc. Suppl.) B42, 96 (1995); K. Kanaya, ibid. B47, 144 (1996).

[6] Y. Iwasaki, K. Kanaya, S. Kaya, S. Sakai, and T. Yoshié, Phys. Rev. D 54, 7010 (1996).

[7] Y. Iwasaki, K. Kanaya, S. Sakai, and T. Yoshié, Nucl. Phys. (Proc. Suppl.) B30, 327 (1993).

[8] M. Bochicchio et al., Nucl. Phys. B262, 331 (1985).

[9] S. Itoh, Y. Iwasaki, Y. Oyanagi, and T. Yoshié, Nucl. Phys. B274, 33 (1986).

[10] Y. Iwasaki, K. Kanaya, S. Sakai, and T. Yoshié, Nucl. Phys. (Proc. Suppl.) B42, 502 (1995); ibid. B47, 515 (1996).

[11] Y. Iwasaki, T. Tsuboi, and T. Yoshié, Phys. Lett. B 220, 602 (1989); Y. Iwasaki, K. Kanaya, S. Sakai, and T. Yoshié, Phys. Rev. Lett. 67, 1494 (1991).

[12] K. Kanaya and S. Kaya, Phys. Rev. D 51, 2404 (1995); P. Buetera and M. Comi, Phys. Rev. B 52, 6185 (1995).

[13] C. DeTar, Nucl. Phys. (Proc. Suppl.) B42, 73 (1995).

[14] L. Maiani and G. Martinelli, Phys. Lett. B 178, 265 (1986); D. Daniel et al., Phys. Rev. D 46, 3130 (1992).

[15] D. Toussaint, Report No. AZPH-TH/96-13, 1996 (to be published).

[16] A. Kocić and J. Kogut, Phys. Rev. Lett. 74, 3112 (1995); Nucl. Phys. B455, 229 (1995). 